\title{
¿Por qué se mueven tanto? Movimiento estudiantil de arquitectura en Buenos Aires (1955-1974)
}

\author{
Why do they move so much? Student movement of \\ architecture in Buenos Aires (1955-1974)
}

\section{Resumen}

Autora:

María Eugenia Durante* durantemariaeugenia@gmail.com

*Universidad Nacional de La Plata
E movimiento estudiantil de arquitectura en Argentina ha llamado la atención de diversos historiadores de las universidades por su magnitud, la profundidad de sus críticas y la construcción de espacios alternativos. Para las décadas de 1960 y 1970, se habla de radicalización política del actor estudiantil debido a que profundiza la vinculación de las prácticas académicas y la militancia política. Para volver sobre estos procesos, este artículo apunta a indagar en la configuración histórica del actor estudiantil en la Facultad de Arquitectura y Urbanismo de la Universidad de Buenos Aires, para comprender cómo fue gestando sus espacios, discursos, prácticas y estrategias. Como fuente principal se recurre a las revistas de arquitectura de la época, las cuales permiten visualizar el impacto de este actor en las instituciones y el discurso público, así como sus diálogos con otros actores de la comunidad universitaria y del campo profesional.

Palabras clave: arquitectura y política; facultades de arquitectura; movimiento estudiantil; Universidad de Buenos Aires; revistas de arquitectura.

\section{Abstract:}

The architecture student movement in Argentina has drawn the attention of several historians from universities for their magnitude, the depth of his criticism and construction of alternative spaces. For the 1960s and 1970s, there is talk of political radicalization of the student actor because it deepens the link between academic practices and political militancy. To return to these processes, this article aims to investigate the historical configuration of the student actor in the Faculty of Architecture and Urbanism of the University of Buenos Aires, to understand how their spaces, discourses, practices and strategies were developed. As the main source, architecture magazines of the time are used, which allow us to visualize the impact of this actor on institutions and public discourse, as well as his dialogues with other actors from the university community and the professional field.

Keywords: architecture and politics; architecture school; student movement; Buenos Aires University; architecture magazines. 


\section{Introducción}

La gran magnitud de la movilización estudiantil en las facultades de arquitectura de Argentina hacia las décadas de 1960 y 1970 ha sido destacada por diversos investigadores de la historia local de las universidades y del movimiento estudiantil en particular. El gran número de estudiantes, docentes y graduados de arquitectura que fueron víctimas del terrorismo de Estado no resulta un dato menor por tratarse de una profesión liberal, cuyo perfil siempre estuvo asociado con las formas de reproducción del capital, la mercantilización del hábitat y los procesos de urbanización. Sin embargo, este perfil hegemónico del profesional de arquitectura encuentra diversos episodios, a lo largo de todo el siglo XX, donde se lo cuestionó profundamente. ¿Arquitectura para qué? ¿para quiénes? Resultan preguntas que emergen de manera recurrente en los sectores que se vinculan a proyectos políticos revolucionarios y/o a procesos de organización social y política que interpelan el orden dominante. En diversos momentos de la historia, la coyuntura política interpeló a los estudiantes y profesionales universitarios y motivó la reconfiguración de las prácticas profesionales proyectándose sobre la lucha política.

En Argentina, a las prácticas de arquitectura que se vincularon a la lucha política se las suele ubicar hacia finales de la década de 1960 y durante los primeros años de 1970. En aquellos años, se profundizó el entrecruzamiento entre el proceso de modernización y el de politización de las capas medias intelectuales de la arquitectura en Argentina. La modernización refiere al proceso de renovación de las instituciones y discursos que apuntó a atender las nuevas demandas del modelo de producción y de las políticas estatales. Este proceso de modernización disciplinar y profesional incorporó temas sociales como incumbencias propias, ante la demanda del Estado de formar expertos para desarrollar nuevas políticas sociales que satisfagan las demandas de vivienda e infraestructura ante la aceleración del proceso de urbanización. Esta impronta social signa el proceso de recepción y adaptación local de las propuestas del proyecto moderno en arquitectura, el cual se convirtió en un horizonte de sentido para quienes buscaban generar respuestas masivas que satisfagan tanto las demandas de la industria de la construcción, como de las políticas públicas.

En Argentina, la adopción de las ideas del proyecto moderno comienza desde la década de 1920 (Brito y Maur, 1993; Longoni y Fonseca, 2010; Molina y Vedia, 2018). Entre las primeras expresiones de la arquitectura moderna, según Brito y Maur (1993), dos posiciones se bifurcan: entre quienes apuntaban a la renovación de la estética formal, y quienes entendían a la arquitectura como parte de una cultura transformadora de la vida, que perseguían la consumación de la utopía del progreso. La arquitectura moderna se consolida hacia la década de 1950 como modelo indiscutible para representar la obra pública y permea en todas las facultades de arquitectura del país, cuya primera experiencia sucede en la de la Universidad Nacional de Tucumán en los años cuarenta.

Hacia la década del 1960, este proceso de modernización se articuló con la radicalización política de los estudiantes y profesionales de arquitectura que se vincularon a la lucha política del sector obrero y las organizaciones de pobladores de villas y asentamientos. La politización operó en la impronta social que le imprimía el problema de la vivienda a la profesión, radicalizando sus discursos y articulando con fuerza la práctica arquitectónica con la práctica militante. Un proceso de creciente politización que sucedió en muchas de las carreras universitarias para aquellos años, y que ha sido largamente estudiado. Una de las tesis más difundidas es la que sostiene que la creciente politización de los ámbitos académicos y profesionales desencadenó en la pérdida paulatina de la autonomía del campo específico, polémica que emerge con la producción de Silvia Sigal (2002). Según esta hipótesis, la centralidad de la política en la vida cotidiana de los sectores medios irrumpió el proceso de modernización y el desarrollo académico y científico desatado desde la caída del peronismo en 1955. A diferencia de estos planteos, para el caso de arquitectura se encuentra que la politización no operó como ruptura del proceso de modernización, sino como recuperación y re-actualización de algunos de sus discursos originales. Autores como Malecki (2016), hablan de una aceleración de los impulsos modernizadores ante la radicalización en arquitectura, mientras que Jajamovich (2014) sostiene que las relaciones entre las prácticas militantes y académicas dan cuenta de múltiples y complejas relaciones, en los que las teorías de la pérdida de autonomía se matizan.

Las prácticas profesionales que se vincularon a los procesos de radicalización política han motivado el debate en torno a la relación entre la técnica y la política, entre trabajo profesional y trabajo militante. En este sentido, estudios recientes de Rigotti (2018), sentencian diferencias entre los expertos, aquellos cuya práctica sistemática se abocó a resolver la política pública, y los vanguardistas, quienes se subsumieron en un discurso comprometido con la lucha política, dos figuras que emergen para diferenciarse del profesional liberal. Los trabajos de Carranza (2014) y Silvestri (2014) se refieren al pasaje del profesional modernizador al profesional comprometido para dar cuenta de lo que sucede hacia la década 1960 -lo cual vinculan a la obra de Sartre-, mientras que para 1970, se habla de paso hacia la idea del intelectual orgánico retomando influencias de Gramsci. Aportando a estos debates, este artículo profundiza en la configuración histórica de las particularidades que adquieren la relación entre las prácticas académicas y las prácticas militantes de un sector del movimiento estudiantil de arquitectura. 


\section{Aspectos metodológicos y fuentes}

Este artículo apunta a caracterizar las diversas y singulares formas de organización, movilización y prácticas políticas de un sector del movimiento estudiantil gestado en el seno de la Facultad de Arquitectura y Urbanismo (en adelante FAU) de la Universidad de Buenos Aires (en adelante UBA), actualmente conocida como Facultad de Arquitectura, Diseño y Urbanismo (FADU), al incorporarse las carreras de diseño desde 1983. Este trabajo forma parte de un reciente trabajo de tesis doctoral (Durante, 2020). El artículo comienza con un breve recorrido desde los orígenes de la FAU, para luego profundizar sobre el período que va del golpe a Perón en 1955 a 1974, cuando inician la persecución al movimiento estudiantil de parte de las fuerzas represivas.

Este trabajo se desarrolla a partir de una metodología cualitativa que se basa en el análisis histórico-crítico de fuentes documentales que dan cuenta de los discursos y prácticas del movimiento estudiantil de la FAU. Principalmente, se recurre a las revistas de arquitectura y documentos de la época que permiten realizar una construcción general del período, divisar diversos ámbitos y actores. Se partió de una recolección, análisis y sistematización de los materiales, para luego seleccionar los que presentaban aportes significativos para caracterizar la configuración y cambios que transita el actor estudiantil.

Las revistas especializadas son uno de los materiales más utilizados, debido a que constituyen un reflejo de las discusiones de la época, con maneras particulares de relatarlas e interpretarlas. No se consideran documentos objetivos, ni relatos fehacientes de lo sucedido, sino un medio para caracterizar los procesos y divisar miradas particulares de los actores, atravesado por disputas e intereses entre quienes deciden qué publicar, qué decir condicionados por los recursos, agentes publicitarios y las instituciones que representan. Las revistas de arquitectura se fueron constituyendo en plataformas que permiten legitimar, en el discurso público, prácticas y saberes sobre el ejercicio profesional. En este sentido, encontrar vestigios de los cuestionamientos a la profesión en medios hegemónicos del campo, da cuenta de la magnitud que tomaron estas discusiones en sus contextos.

A la vez, diversos trabajos recientes se constituyen en fuentes secundarias de este artículo. El caso de la FAU está presente en los trabajos de Bonavena (2005) y Seia (2018), quienes revisan sus formas de organización y participación política en relación al proceso general que protagonizaba el movimiento estudiantil en Argentina. Por otro lado, el documental "70 y pico" (Corbacho, 2016) se constituyó en una referencia importante desde la cual volver sobre aquel proceso silenciado por el golpe militar. Su director, Corbacho, junto a Díaz (2014) han profundizado sobre una de las organizaciones estudiantiles que protagoniza el debate de la época, la Tendencia universitaria popular de arquitectura y urbanismo (TUPAU). Este aporte se suma a los trabajos de Ana Cravino (2015 y 2018) quien reconstruye los conflictos y debates en el seno del movimiento estudiantil y entre los docentes de la FAU desde el golpe a Perón en 1955 hasta la década de 1970.

\section{Resultados. El movimiento estudiantil en la FAU}

El caso del movimiento estudiantil en la FAU permite divisar un proceso particular que se entrelaza a los debates más generales del campo profesional y disciplinar, donde indagar en las singularidades de los estudiantes de arquitectura. Los resultados arrojados por la investigación se presentan en tres apartados que recorren las particularidades de tres períodos históricos. Una primera parte, que indaga en los antecedentes previos al período de estudio en Buenos Aires, buscando las primeras formas de organización y discursos disidentes de los estudiantes de arquitectura. Una segunda parte que relata las formas que emergen previo a la intervención de la noche de los bastones largos, en 1966; y una tercera, que profundiza sobre las formas posteriores.

\subsection{Resistencia a las tradiciones (1910-1955)}

La carrera de arquitectura en la UBA es la primera de país, con su primer graduado en 1878; se constituyó en Escuela en 1901 y se transformó en Facultad en 1948 Durante la primer mitad de siglo XX, en la UBA se gestó la primera generación de profesionales locales debido a que pasaron varios años hasta que se multiplicó la carrera en otras partes del país ${ }^{1}$. Desde sus inicios, la carrera propuso una formación basada en el modelo Beaux-arts que acentuaba el trabajo de la composición y el lenguaje clásico, implementado con el plan de estudios de 1901; una formación de arquitectos-artistas que caracterizó las primeras cuatro décadas del siglo $\mathrm{XX}$ en todas las escuelas de arquitectura del país. Los primeros graduados apuntaron a la constitución de campo profesional de la arquitectura argentina, lo que implicó un proceso de definición de los saberes, prácticas, instituciones, mecanismos de legitimación y regulación propios (Cirvini, 2003). En este proceso, la Sociedad Central de Arquitectos (en adelante SCA), se alzó como la principal institución que representaba los intereses del sector. La SCA se creó en 1886 y su primera propuesta para regular la profesión data de 1904, a la que le siguieron otras tantas que no lograron efectivizar (Brandariz, 1991, p. 37). Recién en 1944 el General Farrel estableció por decreto las normas que regularon el ejercicio de los arquitectos.

Desde los años veinte, los estudiantes cuestionaron y se resistieron al plantel docente debido a su perfil conservador y tradicionalista. Los estudiantes pugnaban por la renovación de la Escuela y "hacían causa común en lugares como el Centro de Estudiantes y desde allí

Las otras universidades públicas en sumar arquitectura serán: Santa Fe en 1923 (luego se dividirá en la Universidad de Rosario Facultad 1971- y del Litoral -Facultad en 1985); Córdoba en 1936 (transformada en Facultad en 1954); Tucumán en 1939 (Facultad en 1952); San Juan en 1950 (Facultad en 1983); La Plata en 1952 (Facultad en 1956); Chaco en 1956 (Facultad en 1973); Mar del Plata en 1962 (Facultad en 1974). (Durante, 2020). 
presionaban no solo para que se modificara el plan de estudios sino también para invitar al país a arquitectos extranjeros que representaran esas nuevas ideas" (Williams, 2018, p. 25). En los años cuarenta, se acrecentó la crítica a la formación debido a que contrastaba con la arquitectura moderna que difundían las revistas especializadas de la época. Para los estudiantes, el sistema Beaux-Arts "ya no les resultaba válido para afrontar las soluciones que la arquitectura debía proveer en la sociedad contemporánea" (Schmit, Silvestri y Rojas, 2004, p.36). El Centro de Estudiantes de Arquitectura (en adelante CEA) fue fundado en 1908, y rápidamente se constituyó "no solo como núcleo de sociabilidad y cuna de futuras asociaciones entre los estudiantes sino también como plataforma desde la cual estos se posicionaban políticamente" (Williams, 2018, p. 25).

En 1946, el gobierno de Perón intervino la Universidad por lo que suceden años conflictivos y contradictorios. En la FAU, amplios sectores cuestionaron las políticas del peronismo, sin embargo, durante aquellos años se generaron una serie de reformas en las escuelas de arquitectura que modernizaron su formación, y varias de ellas se transformaron en facultades "concretándose así la plena autonomía de la disciplina respecto de otras técnicas y artes de la construcción" (Méndez Mosquera, 2018, p. 30). Al inicio del gobierno de Perón, en 1946, la carrera de arquitectura se impartía en cuatro universidades del país, mientras que para 1952 eran seis y algunas lograron jerarquizarse. La puesta en marcha de la FAU, en 1948 no implicó modificaciones de su plantel docente. La renovación comenzó lentamente a través de profesores visitantes o interinos y la puesta en marcha de cursos paralelos y grupos de estudio por fuera de la Universidad. Recién para 1955 se renovó por completo la enseñanza con la adopción plena del movimiento moderno, no sin conflictos y complicaciones (Batlle, 2018).

Durante el primer peronismo, la relación entre la comunidad universitaria y el gobierno tuvo diversas complejidades y se tensó hacia fines del segundo período. Para 1952, se profundizó la injerencia del Poder Ejecutivo en las decisiones universitarias, lo cual es rechazado con fuerza por el movimiento estudiantil, que alzaba las históricas banderas reformistas en defensa de la autonomía y el co-gobierno. Sobre el gremio estudiantil, se instauró la elección de delegados estudiantiles por sorteo entre los mejores estudiantes del último año (Cravino, 2015) y hacia los años cincuenta se exigió que dichos representantes provengan de agrupaciones avaladas por el mismo gobierno. Estas medidas llevaron al CEA a funcionar en un local por fuera de la facultad, a la vez que se multiplicaron los grupos extra-universitarios de estudio y formación. Las relaciones se tensaron hacia fines de 1954 con una huelga universitaria que culminó con varios estudiantes presos. Ese mismo año, se realizó en Córdoba la Convención Nacional de Estudiantes de Arquitectura, uno de los primeros encuentros que convocó a los Centros de Estudiantes de todas las escuelas y facultades del país. Estos hechos muestran el crecimiento de la organización del sector estudiantil de arquitectura y sus primeras redes en el país.

A pesar de la censura, el CEA siguió funcionando y construyendo su legitimidad durante el gobierno de Perón. Desde diferentes medios especializados ${ }^{2}$ el Centro alzó su voz publicando artículos que consideraba importantes para la formación y trabajos de estudiantes. En 1953 comenzó a publicar en la revista Nuestra Arquitectura (en adelante $\mathrm{NA})^{3}$, en las páginas que le cedió su dirección. Entre las primeras publicaciones, el presidente del CEA, Carlos Carabelli, sostenía que la misión del Centro superaba la misión universitaria, persiguiendo fines que suplían a la misma: "uno de los fines de la universidad es formar técnicos y suponemos que en un momento determinado la enseñanza de la universidad es pobre, inadecuada o simplemente inútil; el centro, en este caso, debería organizar cursos para suplir esta deficiencia" (Carabelli, 1954, p.93). En estas expresiones se revela la capacidad de iniciativa que desarrollan ámbitos como el CEA, que se entendía en la misión de construir los espacios que la Universidad no provea: "Evidentemente, si el centro edita textos es porque no lo hace quien debería hacerlo; organiza concursos porque no lo hace la universidad, sienta
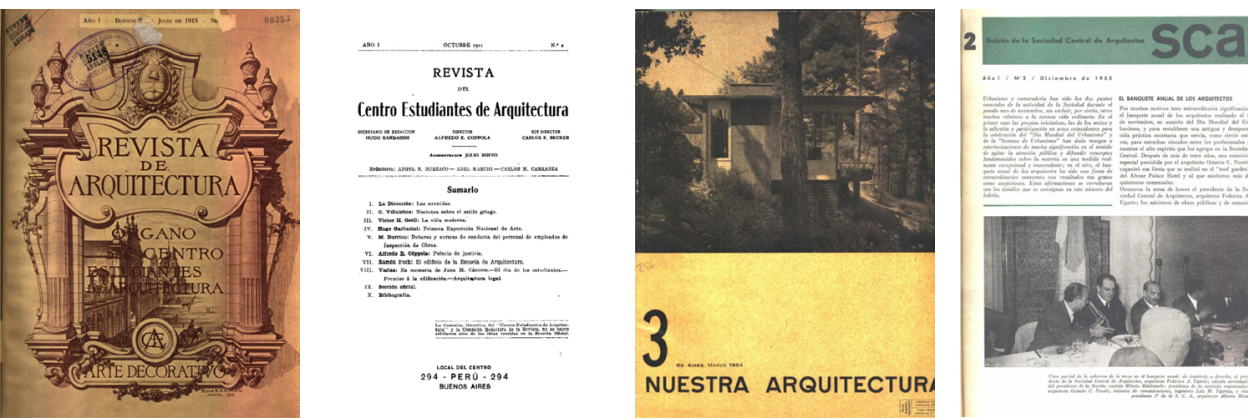

Figura 1: Portada Revista de Arquitectura Nro. 1. Figura 2: Portada Revista del Centro de Estudiantes de Arquitectura Nro. 2. Figura 3: Portada Revista Nuestra Arquitectura Nro. 296. Figura 4: Portada Boletín Sociedad Central de Arquitectos Nro. 2

Fuente: Centro de Documentación-Biblioteca "Prof. Arq. Manuel Ignacio Net", FADU-UBA, Buenos Aires, Argentina

2 Siguiendo el trabajo de Cirvini (2003), el CEA tuvo una primera revista propia entre 1911 y 1912, luego gestó la Revista de Arquitectura en 1915, hasta que en 1917 se acopló la SCA a la misma. En 1951 el CEA se retiró de esta revista y quedó al frente la SCA. En 1953, la revista Nuestra Arquitectura abrió unas páginas a la participación del CEA, y luego de 1955, hizo lo mismo el Boletín de la SCA.
${ }^{3}$ Entre 1915 y 1955 las revistas de mayor continuidad y presencia en el campo profesional serán Nuestra Arquitectura (NA) y la Revista de Arquitectura de la SCA. NA fue una de las revistas con mayor continuidad, trayectoria e influencia dentro del campo profesional, se fundó en 1929 y publicó 523 números de la misma, sin interrupciones 
posiciones frente a determinados hechos porque no lo hace la universidad" (Carabelli, 1954, p. 93). Los estudiantes se alzaban en la responsabilidad de moldear el futuro, su horizonte de sentido se extendía más allá de su tránsito como estudiantes y en conexión con lo que sucedía fuera de la Universidad, donde entusiasmaba la adopción de la arquitectura moderna por considerarla en sintonía con esa mirada joven y prospectiva.

\subsection{La modernización de las formas organiza- tivas (1955-1966)}

Con la caída del peronismo, se abre un período de gran inestabilidad política con la sucesión de gobiernos elegidos democráticamente y golpes militares, y un proceso creciente de conflictividad social, de constitución de nuevos actores y reubicación de otros en el campo de la lucha política. En este marco, en la Universidad hubo un fuerte reclamo por la incorporación de las problemáticas de la realidad social a la formación y, en paralelo, las instituciones profesionales pugnaron por la legitimación de las prácticas ligadas a la cuestión social. Se desarrolló un clima complejo con el peronismo proscripto -entre 1955 y 1973- y las diferencias que surgieron a lo interno de las fuerzas que impulsaron la Revolución Libertadora para destituir a Perón.

Ante la instauración de la Revolución Libertadora, los estudiantes nucleados en la Federación Universitaria Argentina realizaron una toma de las facultades para exigir la renovación de las mismas. Como resultado de la presión, el gobierno nombró como rector de la UBA al candidato propuesto por los estudiantes, José Luis Romero. Sin embargo, también se designó a Atilio Dell'Oro Maini al frente del Ministerio de Educación, quien estaba más vinculado a la Iglesia y a los grupos nacionalistas. Esto último permitió el avance de las gestiones para una nueva Ley de Educación Superior cuyo artículo 28 habilitaba a las universidades privadas. La Ley fue finalmente aprobada en el gobierno de Arturo Frondizi (1958-1962) y permitió la apertura de carreras de arquitectura en instituciones privadas. Durante este período la UBA fue presidida por Risieri Frondizi, quien impulsó concursos docentes, creó nuevos institutos de investigación y facultades, una editorial propia e impulsó la construcción de la ciudad universitaria, entre otras acciones que eran parte del modelo desarrollista. Este proceso de modernización implicó un cambio en las estructuras decisionales, produciéndose reformas de los estatutos de la Universidad que permitieron más espacios de voz y voto a los estudiantes (Califa, 2014, p. 99).

En 1955, la FAU fue ocupada por los estudiantes, quienes realizaron asambleas masivas donde discutieron sobre los rumbos a seguir. Se sucedieron diversas reuniones y jornadas de trabajo, donde se propuso la investigación sobre la situación en otras facultades de arquitectura de la región. Para ello se armaron grupos de estudiantes que indagaron en la situación de los diversos países y

hasta 1986. Por su parte, la Revista de Arquitectura fue creada por el CEA de la FAU-UBA en 1915 y se sumó la SCA en 1917. La revista apareció hasta 1962 ininterrumpidamente (Cirvini, 2003, p. 52), reaparece en 1967 y se mantiene, con alternancias, hasta la actualidad. buscaron experiencias que sirvieran para pensar las posibles reformas a realizar en el plan de estudios y sus contenidos. Uno de los mecanismos importados desde Uruguay, fueron los talleres verticales que se impusieron como principal dispositivo didáctico, donde se pretendía integrar los conocimientos de las materias "teóricas" en el desarrollo de un ejercicio proyectual, el cual a la vez encontraba a estudiantes y docentes de diversos años en diálogo. A la vez, se sumó el aporte de diversas disciplinas humanísticas y la renovación del plantel docente, lo cual permitió realizar cambios de las estructuras y los contenidos.

A la activa participación de los estudiantes se incorporan los jóvenes graduados, un sector novedoso en la arena política universitaria. Este sector era producto del incremento de la matrícula en la facultad y fruto de una generación que se gesta en la discusión de la política universitaria durante el peronismo, el cual, una vez que se graduó, decidió volver sobre la formación para transformarla y constituirse en su nuevo plante docente. A esto se sumó la situación de inestabilidad y falta de oportunidades laborales que comenzó a sentirse con el aumento de los profesionales, diversos factores que alimentaron su reorganización y movilización. Este sector tuvo una participación activa tanto en los debates universitarios como en los del campo profesional a partir de agrupamientos que serán laborales, de estudios, académicos y gremiales, como el Cuerpo de Graduados, creado en 1956 por un espectro de docentes progresistas de la FAU. En esta agrupación participaron varios de los arquitectos preocupados por la relación entre la formación y las problemáticas de la realidad social, que protagonizarán varias de las iniciativas alternativas de formación y trabajo desarrolladas hacia fines de la
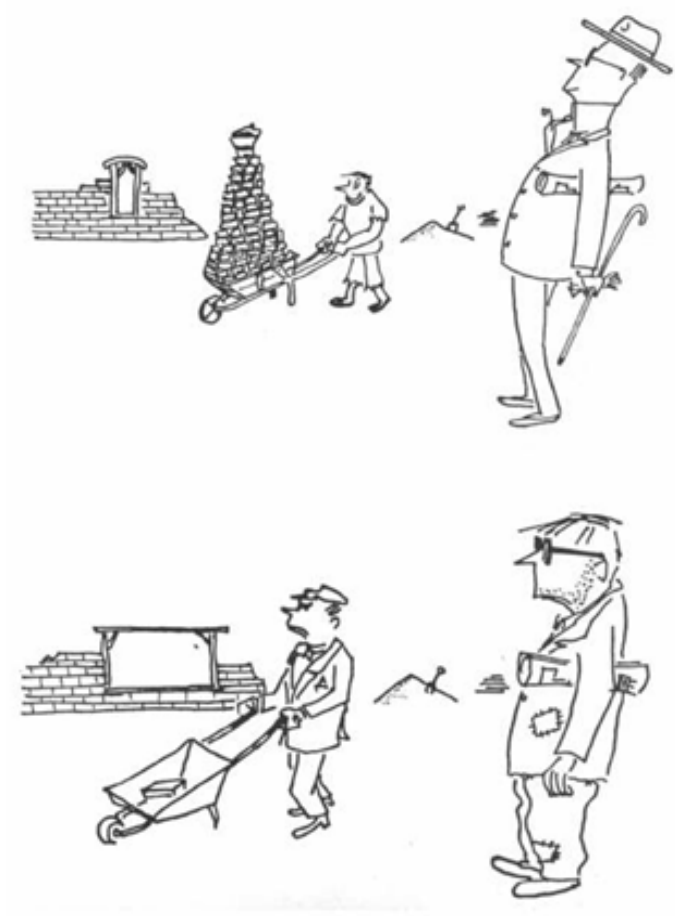

Figura 5: Viñeta publicada en la Revista Nuestra Arquitectura firmada por Hormiga Negra Fuentes: Hormiga Negra (1959) 
El CEA, por su parte, abrió la discusión interna y convocó a docentes a discutir sobre los rumbos de la enseñanza. En septiembre de 1956, las páginas del CEA en NA son firmadas por la nueva conducción, la Agrupación Reformista de Arquitectura (ARA). En sus primeras notas, señalaban que se debía atender a los problemas de la arquitectura (didácticos, gremiales y de contenido), los problemas de la universidad y los problemas del país que se relacionan con la profesión. Entre estos, señalaban al déficit de viviendas como el más grave, ante lo que exigían crear institutos de vivienda y planeamiento regional en cada una de las universidades, garantizando la participación estudiantil para contar con "el empuje progresista e intransigente de la juventud" (CEA, 1956, p.65). Los estudiantes se entendían protagonistas de la época y capaces de motorizar las acciones necesarias para cambiar las condiciones que se presentaban. Expresaban que "Los problemas están, existen, son reales y esperan SOLUCIONES. Y nosotros tenemos mucho que decir. No podemos escribir ahora sobre historia. En este momento tenemos que hacerla" (CEA, 1956, p. 65). A finales de 1959, en las páginas del Boletín de la SCA escribían sobre la "angustia del querer ser" que sufrían los jóvenes estudiantes y arquitectos, y "La sensación de sentirse inútil. Seis años proyectando en un pretendido 'taller', jamás han visto a la idea creadora apoderarse de la materia y tomar forma. Seis años hablando del hombre y no saber cómo tratar a los obreros" (F. J. M., 1959, p. 36).

El creciente protagonismo del sector estudiantil fue una constante de este período, los cuales se agruparon para repensar la formación y la profesión, y vincularon sus discusiones a un proyecto de país y de sociedad que atendiera las problemáticas sociales más urgentes. Algunos sectores se agruparon a partir de determinados partidos políticos y otras veces debido a compartir perspectivas ideológicas, disciplinares y proyectos de sociedad no partidarios. En sus expresiones se hacía visible la confianza que depositaban sobre la arquitectura para aportar a horizontes transformadores, lo cua se relacionaba y entraba en tensión con la necesidad material de buscar nuevos espacios laborales ante el crecimiento de la matrícula.

En este contexto, se desarrolló una de las experiencias más importante de extensión universitaria de la UBA

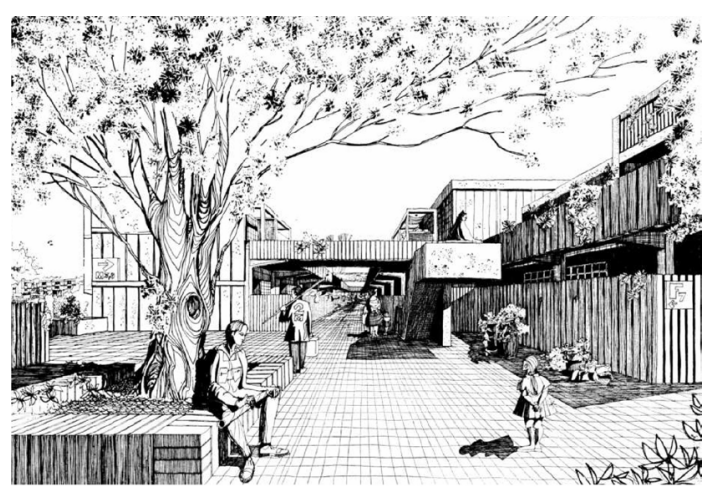

en Isla Maciel; una propuesta que, por primera vez, abordó el problema de la vivienda fuera de las aulas. El Departamento de Extensión Universitaria (DEU) de la UBA se creó en 1956 y comenzó a desarrollar una acción sistemática e interdisciplinaria con los pobladores de la Isla. Las acciones apuntaron a dos aspectos: facilitar el acceso a la Universidad de sectores más amplios de la sociedad, y lograr una reestructuración de la Universidad para que responda a las necesidades reales del país, acompañada de "un cambio de actitud profesional y humana de estudiantes, profesores y egresados" (DEU, 1957, p. 5). Varios de los talleres de la FAU se vincularon a las acciones de la DEU en Isla Maciel hacia 1960. Entre otros, participaron el taller de Wladimiro Acosta y el de Horacio Berretta quienes propusieron a sus estudiantes que proyectaran viviendas para los pobladores. Algunas de estas propuestas fueron publicadas en la Revista Summa y NA. Estas experiencias de extensión universitaria profundizaron el aprendizaje desde la vivencia propia de la realidad social y marcan los inicios de un trabajo territorial donde se resignifica el hecho de "poner el cuerpo", la charla con los usuarios y el saber co-construido.

\subsection{Radicalización política y construcción de alternativas (1966-1974)}

El proceso de modernización y desarrollo científico de la Universidad fue abruptamente interrumpido en julio de 1966, cuando el gobierno de Onganía suprime por ley la autonomía universitaria. Muchos estudiantes y docentes resistieron esta medida y se manifestaron en contra, ocupando diversas facultades. El 29 de julio, la policía ingresa en las facultades y reprime a los manifestantes en lo que se denominó La noche de los bastones largos. La intervención en la UBA implicó un desmantelamiento de los procesos que se venían construyendo previamente, la renuncia masiva de profesores en todas las facultades y un reclamo creciente que profundizó la participación política de los estudiantes. Para el gobierno de Onganía la comunidad universitaria era un peligro por ser considerada un foco del comunismo y las ideas del marxismo.

En la intervención de la FAU fueron desalojados los pabellones, golpeando a estudiantes y docentes. Estos sucesos generaron posiciones diversas entre los

Figura 6: Perspectiva proyecto para Isla Maciel de estudiantes del taller de Horacio Berretta. Figura 7: Perspectiva proyecto Wladimiro Acosta y equipo

Fuentes: Nuestra Arquitectura (1965) y Acosta (1967) 
profesores, "algunos se quedan en sus cargos, aunque condenando la violenta represión ejercida contra alumnos y profesores, otros renuncian con diferente grado de beligerancia y un último grupo permanece en silencio" (Cravino, 2012, p. 5). Luego de varias reuniones, donde se discutía si la renuncia era una estrategia acorde o había que quedarse a resistir el proceso, renunciaron 234 profesores, resultando una de las facultades donde más se sintió la desvinculación de su planta docente. A diferencia de otras profesiones donde varios profesores se exiliaron, gran parte de los de arquitectura se recluyeron en sus actividades profesionales o brindaron clases en sus casas, armaron grupos de estudio y otras formas de organización por fuera de la institución (Cravino, 2012). Ejemplo de esto es el Centro de Estudios del Hábitat, creado en 1967, donde participaron quienes se habían alejado de la FAU en 1966, un espacio por fuera de la institución que se entendía disputando sentidos con la formación universitaria (Summa, 1968).

En este clima, en octubre de 1969, se desarrolló el X Congreso de la Unión Internacional de Arquitectos (UIA) en Buenos Aires. El encuentro convocó a gran cantidad de profesionales y se realizó en paralelo al encuentro de estudiantes-el cual era una constante desde el Congreso de la UIA en Cuba, en 1963. En el encuentro de Buenos Aires se generó una importante ruptura debido a que un grupo de estudiantes irrumpió en el acto de inauguración y exigió que se replantee el temario y forma del congreso. Los estudiantes se hicieron escuchar en el principal encuentro de arquitectos del mundo y, debido al rechazo de las autoridades, organizaron un concurrido encuentro paralelo en la FAU (Carranza, 2011). Un episodio que muestra cómo el movimiento estudiantil profundizó sus reclamos rompiendo con las instituciones tradicionales y avanzando en la construcción de espacios propios.
Para 1971, el gobierno del General Lanusse buscó construir una política universitaria más conciliadora que calle las críticas. Sin embargo, las movilizaciones estudiantiles y tomas de facultades se multiplicaron junto con las intervenciones de las fuerzas de seguridad. En ese contexto, los sectores de izquierda y peronistas cuestionaron la tradición de organización a través de los centros de estudiantes e impulsaron los cuerpos de delegados y asambleas. El cuestionamiento a las instituciones se extendía hacia las formas del sistema representativo estudiantil, generando procesos de "doble poder" (Bonavena, 2005). El cuerpo de delegados buscó instaurarse como poder alternativo a las autoridades $y$ centros de estudiantes, llegando a formular cátedras y seminarios paralelos.

En la FAU, el cuerpo de delegados se formó en el primer cuatrimestre de 1971. En agosto del mismo año, organizaron junto a docentes el "Encuentro Estudiantil/ Docente de Arquitectura" en la ciudad universitaria, que contó con la participación de cerca de mil quinientas personas. En el encuentro, se leyó una carta enviada por el arquitecto Mario Soto desde la cárcel de Villa Devoto. La carta fue considerada una expresión representativa del compromiso político de los arquitectos de la época. Allí Soto afirmaba que "mi condición de arquitecto está íntimamente ligada a mi encarcelamiento" (Soto, 1971, p. 20). A la vez, durante el encuentro se analizaron las experiencias de formación alternativa que se desarrollaban en Córdoba, con el Taller Total (desde 1970), en Rosario, La Plata y en otras facultades de arquitectura del país. Días después del encuentro, los estudiantes levantaron los primeros cursos paralelos, como sucedía en la Facultad de Filosofía y Letras de la UBA. En este escenario, las autoridades decretaron el cierre de la facultad y dan por finalizado el ciclo lectivo, lo que generó una nueva ola de movilizaciones.

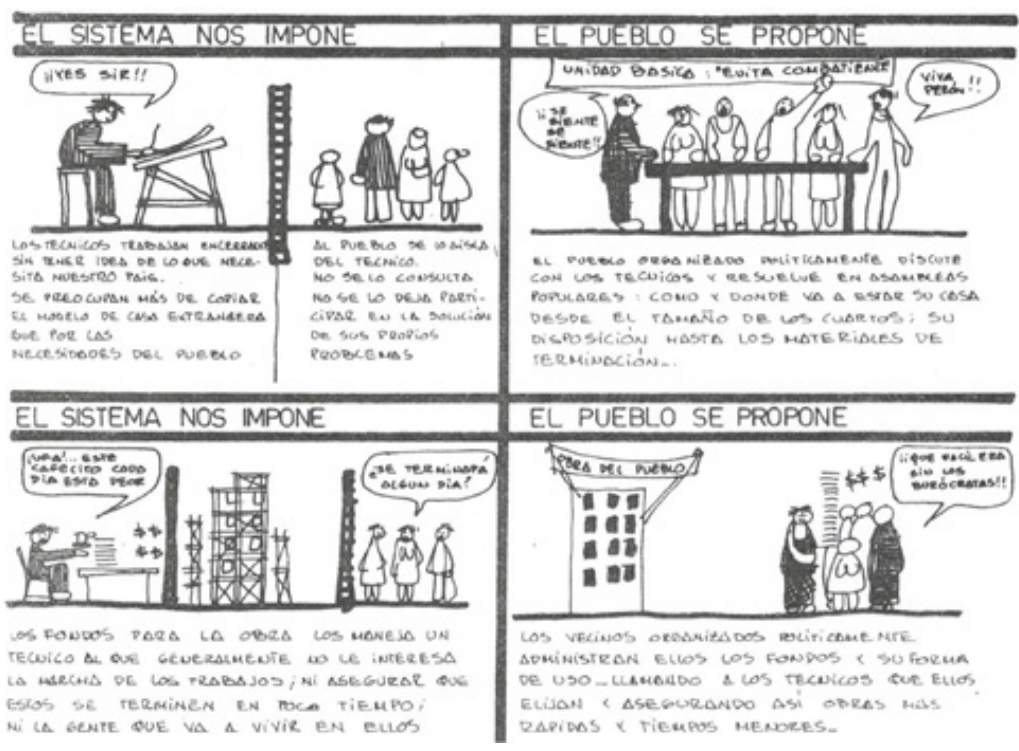

Figura 8: Gráfico cartilla Juventud Universitaria Peronista para el CEA Fuentes: Equipos político-técnicos JUP (1973)

${ }^{4}$ La investigación sobre los congresos de la época merece un desarrollo particular $y$ en profundidad, debido a que se fueron configurando, desde mediados de siglo XX, en las principales arenas de discusión profesional a nivel latinoamericano. Los congresos de la UIA eran de alcance mundial, y su realización en sedes latinoamericanas como Cuba (1963), en Buenos Aires (1969) y en México (1978), se constituyen en tres hitos importantes (Durante, 2020). 
El creciente descontento estudiantil, según Portantiero, era "resultado de la contradicción entre oferta y demanda universitaria, entre las oportunidades de educación superior y los requerimientos de un sistema económico que ofrece escasas perspectivas al trabajo calificado" (1978, p. 15). La crisis de las escuelas de arquitectura se desarrollaba en una situación de crisis laboral y del sector de la construcción que impedía la absorción de un número creciente de graduados en el mercado laboral. Según datos de la UBA, en el año 1950 se graduaron 69 arquitectos, en 1960 fueron 165, en 1970 fueron 325 y en 1975 se graduaron 1328 (Gutiérrez, 1993).

Todos estos episodios que convulsionan el desarrollo de clases en la FAU fueron recuperados por las principales revistas locales de arquitectura, como NA y Summa ${ }^{5}$. A finales de 1971, la revista NA se pregunta por la crisis que se vive en la carrera de arquitectura, "¿La Facultad está en crisis? ¿La arquitectura está en crisis? ¿El país está en crisis?" (Nuestra Arquitectura, 1971, p. 3). Ante esto, realizó entrevistas a diversos protagonistas para "esclarecer el proceso", donde se multiplicaban las críticas a la desconexión de la formación con las problemáticas de la realidad social y señalaban que los contenidos y métodos de enseñanza eran caducos. La revista Summa, por su parte, también realiza una serie de entrevistas que publicaron en su sección titulada "políticas del hábitat". A modo de síntesis, los editores realizan unas conclusiones de las mismas donde señalan dos puntos coincidentes de las diversas expresiones. Primero, la necesidad de una "actitud radical frente a sistema de formación universitaria vigente", y, segundo, que esa actitud debía derivar de "una impugnación total a la estructura socio-económica y política actual, cuyo cambio sustancial se visualiza condición sine qua non para formular un proyecto válido de formación específica para el diseño del hábitat" (Morea et al., 1971, p. 65).
Estos procesos de movilización creciente encontraron en los breves meses de duración del tercer gobierno peronista, con la asunción de Cámpora en mayo de 1973, una posibilidad de institucionalizar varias de sus propuestas para la formación. En este contexto, en la FAU se crea la Federación de Comisiones DocenteEstudiantiles que se encargó de realizar una propuesta político-pedagógica para los cursos de Diseño, que será el antecedente de los Talleres Nacionales y Populares (TANAPO). Estas modificaciones implicaron cambios en las estructuras organizativas y formas de enseñanza, asi como la reconfiguración de los contenidos y enfoque de la disciplina, vinculándola a los problemas sociales. Los talleres se pasaron a nombrar por números en vez de apellidos de profesores, lo que constituía un signo de descrédito construido en torno a la figura del docente, como "gran maestro" y la pérdida de legitimidad de la autoridad tradicional (Cravino, 2018).

Esta breve experiencia de transformación de la carrera fue posible porque se apoyó en los avances teóricos y prácticos que se venían produciendo desde diversos grupos de estudiantes y docentes. Algunos de estos antecedentes fueron los documentos de la organización estudiantil Tendencia Universitaria Popular de Arquitectura y Urbanismo (TUPAU), publicados desde 1967, o las cartillas de los Equipos Técnicos de la Juventud Universitaria Peronista, que desarrollaron prácticas junto a los movimientos villeros. A la vez, varios de los docentes y graduados vinculados a los TANAPO formaron parte de las acciones que llevó adelante la Comisión Municipal de la Vivienda en las villas de la Ciudad de Buenos Aires. En síntesis, las experiencias que repensaron la FAU de raíz eran producto de la confluencia de diversas generaciones que fueron construyendo marcos teórico-metodológicos y técnico-políticos para configurar un ejercicio profesional y saberes disciplinares que abordaran las problemáticas

\section{REACONDICIONAMIENTO URBANO EN VILLAS DE EMERGENCIA}

\section{A - SOLUCIONES PARA REALO.} A - SOLUCIONES PARA
JAMIENTO INMEDIATO

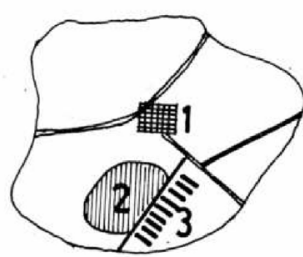

1, Comisión vecinal y equipos Afectada; 3, Realojamiento provisorio.

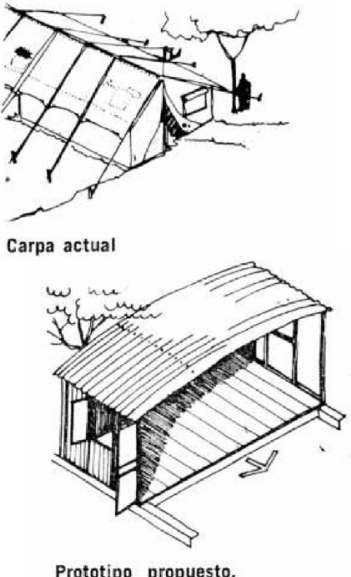

Prototipo propuesto.
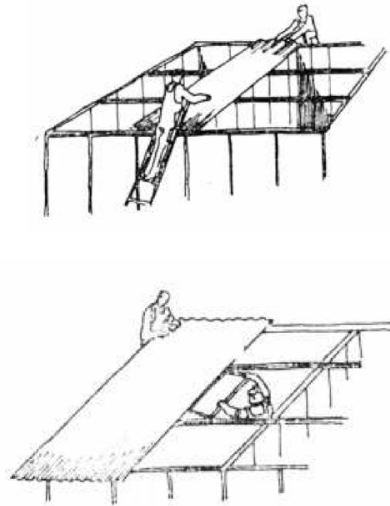

Figura 9: Propuesta para realojamiento en villa de emergencia de la Comisión Municipal de la Vivienda publicado en Nuestra Arquitectura

Fuentes: Nuestra Arquitectura (1974)

${ }^{5}$ La Revista Summa apareció en 1963 y hacia la década de 1970 se

consolidó como una de las revistas más conocidas del campo. 
del hábitat popular, enmarcados en un proyecto político de transformación social. Con la intervención de las universidades de parte del gobierno que se produjo luego de la muerte de Perón en 1974, estas experiencias se ven interrumpidas y muchos de sus protagonistas son perseguidos, detenidos, exiliados y desaparecidos.

\section{Conclusiones finales}

El artículo realiza un rápido recorrido por el proceso de conformación del actor estudiantil y sus formas de organización y movilización en la FAU-UBA. Estos aportes permiten seguir pensando la historia del movimiento estudiantil en el marco de procesos de discusión propios de ciertos ámbitos disciplinares y campos profesionales. Es habitual sesgar la mirada del movimiento estudiantil a los estudiantes en sí y se olvida que los mismos realizan sus trayectos formativos (y de militancia) en tensión con su proyección como futuros profesionales. En este sentido, para enriquecer la mirada sobre los procesos de movilización estudiantil es necesario incorporar la relación de la formación de grado con el campo profesional, sus instituciones, marcos regulatorios, medios de comunicación, discursos públicos, entre muchas otras dimensiones.

Las primeras agrupaciones estudiantiles de la FAU se entendían modelando el incipiente campo profesional, y se pensaban como los encargados de impulsar las renovaciones que requerían las nuevas condiciones sociales y económicas del país. En este sentido, las generaciones que se forman hacia los años '50 profundizan la modernización de sus formas y cuestionan el acotado alcance de sus prácticas, construyendo los primeros abordajes sistemáticos ante las nuevas demandas sociales. Una amplitud demandada por un Estado en crecimiento, pero, a la vez, ante el aumento de los graduados y la presión sobre el mundo laboral. Hacia fines de los años '60 y principios de los '70, la crisis generalizada en diversas esferas de la vida cotidiana y el cuestionamiento a las instituciones impacta en el movimiento y sus perspectivas: ya no consistía en formarse para el contexto que los recibía al graduarse, sino que había que cambiar de raíz la universidad, la profesión y la sociedad.

Este sector estudiantil organizado tenía vínculos con las principales revistas de arquitectura, que le dieron lugar en sus páginas, discutieron con las instituciones y sociedades profesionales sobre el mundo laboral, participaron e irrumpieron en los congresos internacionales de arquitectos. Todas estas acciones muestran a un movimiento que se proyecta sobre los debates propios del campo profesional y disciplinar. En contraste con quienes sostienen que opera una "disolución de la disciplina" ante la radicalización política, se visualiza una preocupación por la construcción de nuevas prácticas y saberes, sin el abandono de sus posiciones como estudiantes y futuros profesionales de arquitectura. De hecho, la crisis creciente del sector de la construcción, por ende, del mercado laboral de los profesionales y la desvinculación de las prácticas con las problemáticas sociales genera cuestionamientos profundos que no siempre implican un entrelazamiento con los partidos políticos; elementos interesantes de sumar a las vastas investigaciones que han reconstruido la historia de las universidades, de la enseñanza, de los intelectuales y del movimiento estudiantil. En este sentido, se entiende que estos aportes buscan volver sobre los vínculos y tensiones que emergen entre las prácticas profesionales y las prácticas militantes, entre el debate disciplinar y el debate político.

\section{Agradecimientos}

Se agradece al Consejo Nacional de Investigaciones Científicas y Técnicas de Argentina (CONICET) por financiar la beca de investigación marco, y al Centro Interdisciplinario de Estudios Complejos (CIEC) de la Facultad de Arquitectura y Urbanismo (FAU) de la Universidad Nacional de La Plata (UNLP) por disponer el espacio de trabajo.

Cómo citar este artículo/How to cite this article: Durante, M.(2021). ¿Por qué se mueven tanto? Movimiento estudiantil de arquitectura en Buenos Aires (1955-1974). Estoa. Revista de la Facultad de Arquitectura y Urbanismo de la Universidad de Cuenca, 10(20), 165 - 175. doi: 10.18537/est. v010.n020.a14 


\section{Referencias bibliográficas}

Acosta, W. (1967). Isla Maciel, Summa, (10), 56-57.

Batlle, S. (2018). Los sesenta. Enseñanza y profesión. En Batlle, S. y Méndez Mosquera, S. (Coord.), De Alumnos y Arquitectos. DAR, FADU-UBA.

Bonavena, P. (2005). Cuerpos de delegados en la Facultad de Arquitectura de la UBA y de la Escuela de Bellas Artes Prilidiano Pueyrredón, Revista Praxis, (1).

Brandariz, G. A. (1991). Breve historia de la profesión de arquitectura en la República Argentina, Consejo Profesional de Arquitectura y Urbanismo, (4), 26-46.

Brito, G. A. y Maur, I. (1993). Buenos Aires 1920-1940 una modernidad silenciosa. En López Rangel, R. (Comp.), La Primera Modernidad Arquitectónica en América Latina (pp. 1-44). Universidad Autónoma Metropolitana de México, Instituto Francés para América Latina.

Califa, J. S. (2014). La socialización política estudiantil en la Argentina de los sesenta, Perfiles educativos, 36 (146), 98-113.

Carabelli, C. (1954). Misión de un Centro de Estudiantes, Nuestra Arquitectura, (296), 93

Carranza, M. (2011). La arquitectura rebelde. E movimiento estudiantil en el X Congreso Mundial de la Unión Internacional de Arquitectos. Buenos Aires, 1969", Conflicto Social, 4 (5), 124-145.

Carranza, M. (2014). Entrelazamientos: Cultura política y cultura del espacio en el VII Congreso Mundial de Arquitectos de la UIA, La Habana, Cuba, 1963, Registros, 10 (11), 40-56

CEA (1953). Páginas del Centro de Estudiantes de Arquitectura, Nuestra Arquitectura, (290), 253

Cirvini, A. (2003). Nosotros los Arquitectos. Campo disciplinar y profesión en la Argentina moderna. Fondo Nacional de las Artes.

Corbacho, M. y Díaz, J. P. (2014). Arquitectura y dependencia. Vida y obra de la TUPAU (tendencia universitaria popular de arquitectura y urbanismo). En $\checkmark$ Jornadas de Estudio y Reflexión sobre el Movimiento Estudiantil Argentino y Latinoamericano.

Cravino, A. (2012). Antecedentes del movimiento estudiantil radicalizado: Una crónica de la situación de la Facultad de Arquitectura de la Universidad de Buenos Aires entre la Noche de los Bastones largos y el Congreso Mundial de Arquitectura. En IV Jornadas de Estudio y Reflexión sobre el Movimiento Estudiantil Argentino y Latinoamericano. http://conflictosocialiigg.sociales.uba. ar/iv-jornadas-movimiento-estudiantil/

Cravino, A. (2015). Nosotros somos la Universidad. En XI Jornadas de Sociología. Facultad de Ciencias Sociales, Universidad de Buenos Aires.
Cravino, A. (2018). Esperando la Revolución: 1966-1974. Revista Movimiento, (5), 89-111.

DEU (1957). 1er año de Extensión Universitaria. Universidad de Buenos Aires.

Durante, M. E. (2020). Historias para una arquitectura militante. Circulación de ideas en Latinoamérica y politización de la arquitectura argentina en los años sesenta y setenta (Tesis doctoral). Universidad Nacional de General Sarmiento.

Equipos político-técnicos JUP (1973). Bases para un proyecto político-técnico de construcciones masivas con participación popular. Centro de Estudiantes FAU-UBA.

F. J. M. (1959). Hablan los estudiantes de arquitectura, Boletín de la SCA, (30), 36-37.

Gutiérrez, R. (1993). Sociedad Central de Arquitectos. 100 años de compromiso con el país: 1886/1986. Sociedad Central de Arquitectos.

Hormiga Negra (1959). Ayer y Hoy, Nuestra Arquitectura, (352), 41.

Jajamovich, G. (2014). Entre la técnica y la política: Mario Corea, su equipo y su propuesta para el Concurso de remodelación del área central de Santiago de Chile (1972), Registros, 10 (11), 98-114.

Longoni, R. y Fonseca, I. (2010). La enseñanza de la Arquitectura y el Urbanismo en el Primer Gobierno peronista. En II Congreso de Estudios sobre el Peronismo 1943-1976. Red de Estudios sobre el Peronismo. Universidad Nacional de Tres de Febrero

Malecki, S. (2016). Crisis, radicalización y política en el Taller Total de Córdoba, 1970-1975. Prohistoria, (25), 79 103.

Méndez Mosquera, S. (2018). Década del 40. De Escuela de Arquitectura a Facultad. En Batlle, S. y Méndez Mosquera, S. (Coord.), De Alumnos y Arquitectos. DAR, FADU-UBA.

Molina y Vedia, J. (2018). El cese de 1955 en la Universidad Nacional de Buenos Aires (UBA). Quiebre de conocimiento clásico. En Batlle, S. y Méndez Mosquera, S. (Coord.), De Alumnos y Arquitectos. DAR, FADU-UBA.

Morea, L., Ursini, C., Mérega, G., Herrero, F., Forni, F. y Palacios Videla, I. (1971). Formación universitaria y transformación del hábitat, Summa, (43), 61-65.

Nuestra Arquitectura (1965). Viviendas para el desarrollo de una comunidad, Nuestra Arquitectura, (423), 44-46.

Nuestra Arquitectura (1971). La crisis en la Facultad de Arquitectura, Nuestra Arquitectura, (474), 8-10.

Nuestra Arquitectura (1974). Mejoramiento en villas: labor de la CMV. Nuestra Arquitectura, (488), 22-25.

Portantiero, J. C. (1978). Estudiantes y política en América Latina. Siglo XXI editores. 
Rigotti, A. M. (2018). Profesionales, expertos y vanguardia: la cultura arquitectónica del Cono Sur: Actas Seminario Internacional. UNR Editora.

Schmidt, C.; Silvestri, G. y Rojas, M. (2004). Enseñanza de arquitectura, en Liernur, J. F. y Aliata, F. (Comp.) Diccionario de arquitectura en la Argentina: estilos, obras, biografías, instituciones, ciudades, (32-44). Clarín Arquitectura.

Seia, G. (2018). De la Revolución a la Reforma. Reconfiguraciones de las formas de militancia estudiantil en la Universidad de Buenos Aires entre 1976 y 1983 (Tesis Doctoral). Facultad de Ciencias Sociales, Universidad de Buenos Aires.

Sigal, S. (2002). Intelectuales y poder en Argentina: Ia década del sesenta. Siglo XXI.

Silvestri, G. (2014). Alma de arquitecto. Conformación histórica del 'habitus' de los proyectistas del hábitat, Registros, 10 (11), 72-97

Soto, M. (1971). Arquitecto Soto: por la construcción de una nueva sociedad, Cristianismo y Revolución, 4 (29), 20.

Summa (1968). Centro de Estudios del Hábitat, Summa (11), 74.

Williams, F. (2018). La Escuela de Arquitectura en los años 30: nuevos desafíos y crisis de la enseñanza. En Batlle, S. y Méndez Mosquera, S. (Coord.), De Alumnos y Arquitectos. DAR, FADU-UBA. 